\title{
Editorial
}

\section{Echocardiographic assessment of long axis function: a simple solution to a complex problem?}

Circumferential fibre shortening has formed the dominant basis for the conventional analysis of left ventricular function by echocardiography with measurement of ventricular dimensions, ejection fraction, and fractional shortening; however, the complexity of ventricular architecture has been recognised by anatomists for almost two centuries. The presence of additional myocardial fibres oriented in the longitudinal direction and located in the subendocardial, trabecular, and papillary muscle regions of the ventricle $^{1}$ has also led to an interest in echocardiographic assessment of the left ventricular long axis function. As there is a constant relation between the cardiac and the chest wall, displacement of the atrioventricular plane towards the apex is a direct reflection of longitudinal fibre contraction. Accurate measurement of this displacement at the anterior, posterior, lateral, and medial aspect of the atrioventricular ring is possible using two dimensionally guided $M$ mode echocardiography from the apical two and four chamber views, allowing information on both regional and global left ventricular function, the latter measured as an average displacement from all four planes. $^{2}$

\section{Long axis function}

Abnormalities in long axis systolic function occur in a variety of pathological conditions including myocardial infarction, ${ }^{2}$ coronary artery disease, ${ }^{3}$ cardiac failure, ${ }^{4}$ and systemic sclerosis. ${ }^{5}$ Functional abnormalities of the ventricular long axis are not limited to reduction in systolic contraction but may manifest as diastolic abnormalities or alterations in the interaction with circumferential short axis fibres. Incoordination between long and short axis function ${ }^{6}$ in ventricular disease can result in asynchrony of left ventricular relaxation and associated filling abnormalities. Long axis shortening usually occurs before any short axis contraction, causing the ventricle to become more spherical during the phase of isovolumetric contraction; however, in ventricular disease, particularly where there is subendocardial ischaemia predominantly affecting the long axis fibres, ventricular shape changes occur resulting in a less efficient and more incoordinated ventricular contraction.?

\section{Long axis function and ejection fraction}

There is a clear relation between long axis function and ejection fraction ${ }^{2-4}$ but atrioventricular plane displacement (AVPD) has a number of potential advantages. It can be obtained from the apical views, which allow satisfactory imaging in the vast majority of patients yet it is not limited by the need for accurate endocardial border identification, notoriously difficult particularly in elderly patients. In addition, AVPD is a highly reproducible measurement even when performed by relatively inexperienced operators. ${ }^{8}$ However, the wealth of data relating ejection fraction to prognosis in left ventricular dysfunction has ensured that this remains the most widely applied echocardiographic assessment of global left ventricular function. An article published in this edition of Heart is the first available evidence that AVPD, in a similar manner to ejection fraction, may also provide prognostic information in patients with significant left ventricular dysfunction. ${ }^{9}$ Although ejection fraction was not measured in this study, the assessment of echo quality highlights the fact that AVPD may be more widely applicable to this patient population being readily obtained in all of the patients studied, whereas two dimensional imaging of sufficient quality to measure ejection fraction was possible in only $77 \%$ of cases. Mortality is significantly higher when the AVPD is less than $10 \mathrm{~mm}$ with a substantial mortality increase when AVPD is less than $7 \mathrm{~mm}$.

The similarity between AVPD and ejection fraction techniques for the assessment of global left ventricular function is readily apparent but they may not produce identical information. Long axis fibres are mainly subendocardial and more prone to ischaemia, implying that abnormalities of AVPD may occur earlier in the disease process and represent more subtle alterations in ventricular function. It may also better reflect global function by deriving information from four distinct ventricular regions and may ultimately prove to be a more accurate prognostic indicator than ejection fraction. The relation between AVPD and prognosis in patients with left ventricular dysfunction requires further confirmation and there remains a lack of data on normal AVPD at different ages and with alterations in posture. Its value for serial investigation remains unclear, however, the excellent reproducibility of the technique tends to suggest that it would be ideally suited to serial application.

\section{AVPD and left ventricular function}

Despite the major advances in cardiac ultrasound image acquisition and analysis in recent years, the conventional assessment of left ventricular function remains subject to many limitations. It requires high definition endocardial border detection-still possible in only a proportion of patients-as well as careful attention to analysis techniques. Echocardiographic assessment of long axis left ventricular function may not provide the complete answer for ventricular function assessment and it is unlikely to displace conventional and well established techniques. With improved image resolution and the prospect of three dimensional echocardiography, accurate measurement of left ventricular function by echocardiographic techniques will continue to improve. However, this reproducible, widely applicable, and potentially important prognostic technique may yet prove the match of more sophisticated function analysis. Its beauty lies in its simplicity.

Wessex Regional Cardiac Unit, IAIN A SIMPSON Southampton University Hospital, Southampton, United Kingdom

1 Greenbaum RA, Ho SY, Gibson DG, Becker AF, Anderson RH. Left ventricular fibre architecture in man. Br Heart $\mathcal{f} 1981 ; 45: 248-63$. 
2 Alam M. The atrioventricular plane displacement as a means of evaluating left ventricular systolic function in acute myocardial infarction. Clin Cardiol 1991;14:588-94.

3 Alam M, Hoglund C, Thorstrand C, Hellekant C. Haemodynamic significance of the atrioventricular plane displacement in patients with coronary cance of the atrioventricular plane displacement

4 Alam M, Hoglund C, Thorstrand C. Longitudinal systolic shortening of the left ventricle: an echocardiographic study in subjects with and withthe left ventricle: an echocardiographic study in subjects with
out perserved global function. Clin Physiol 1992;12:443-52.

5 Henein MY, Cailes J, O'Sullivan C, duBois RM, Gibson DG. Abnormal Hentricular long-axis function in systemic sclerosis. Chest 1995;108:
vention
1533-40.

6 Henein MY, Gibson DG. Suppression of left ventricular diastolic filling by long axis asynchrony. Br Heart $\mathcal{F}$ 1995;73:151-7.

7 Jones CJ, Raposo L. Gibson DG. Functional importance of the long axis dynamics of the human left ventricle. Br Heart f 1990;63:215-20.

8 Alam M, Rosenhamer G. Atrioventricular plane displacement and left ventricular function. F Am Soc Echocardiogr 1992;5:427-33.

9 Willenheimer R, Cline C, Erhardt L, Israelsson B. Left ventricular atrioventricular plane displacement: an echocardiographic technique for rapid assessment of prognosis in heart failure. Heart 1997;78: for rap $230-6$.

\section{STAMPS IN CARDIOLOGY}

\section{Harvey Williams Cushing (1869-1939)}

This 45 cent stamp was issued by the USA on 17 June 1988 as a further addition to the Great Americans series. On the stamp's official first day cover for the American Association of Neurological Surgeons (founded as the Harvey Cushing Society in 1931) it is stated that the stamp was issued "for the work that he did and for his many contributions to the medical profession, and to American society".

Harvey Williams Cushing was a great neurosurgeon, famous especially for his surgery of the pituitary gland and for his description of the syndrome caused by a basophil adenoma of the gland. Cushing's syndrome causes serious heart disease, chiefly through hypertension and coronary atheroma as well as the effects of an excess of cortisol, aldosterone, and androgens. Congestive heart failure occurs in $25 \%$ and its poor response to treatment has led to the syndrome being called a killer disease. In 1908 Cushing carried out pioneer work in experimental cardiac surgery (Fournal of Medical Research 1908;17:471-85). $\mathrm{He}$ was the first to produce valve lesions (in dogs) systematically rather than by blind instrumentation. Using a valvulotome introduced into the apex of the ventricle he produced mitral and tricuspid regurgitation by cutting the leaflets, and he also created stenosis of the valves by tying a ligature around the 